\title{
Randomised controlled study of clinical outcome following trophic feeding
}

\author{
R J McClure, Simon J Newell
}

\begin{abstract}
Aims-To determine the effect of trophic feeding on clinical outcome in ill preterm infants.

Methods-A randomised, controlled, prospective study of 100 preterm infants, weighing less than $1750 \mathrm{~g}$ at birth and requiring ventilatory support and parenteral nutrition, was performed. Group TF (48 infants) received trophic feeding from day $3(0.5-1 \mathrm{ml} / \mathrm{h})$ along with parenteral nutrition until ventilatory support finished. Group C (52 infants) received parenteral nutrition alone. "Nutritive" milk feeding was then introduced to both groups. Clinical outcomes measured included total energy intake and growth over the first six postnatal weeks, sepsis incidence, liver function, milk tolerance, duration of respiratory support, duration of hospital stay and complication incidence.
\end{abstract}

Results-Groups were well matched for birthweight, gestation and CRIB scores. Infants in group TF had significantly greater energy intake, mean difference 41.4 (95\% confidence interval $9,73.7)$ kcal/kg p=0.02; weight gain, 130 (CI 1, 250) g $\mathbf{p}=0.02$; head circumference gain, mean difference 0.7 (CI $0.1,1.3) \mathrm{cm}, \mathrm{p}=0.04$; fewer episodes of culture confirmed sepsis, mean difference $-0.7(-1.3,-0.2)$ episodes, $p=0.04$; less parenteral nutrition, mean difference -11.5 (CI $-20,-3)$ days, $p$ $=0.03$; tolerated full milk feeds $(165 \mathrm{ml} / \mathrm{kg} /$ day) earlier, mean difference -11.2 (CI $-19,-3)$ days, $p=0.03$; reduced requirement for supplemental oxygen, mean difference $-22.4(\mathrm{CI}-41.5,-3.3)$ days, $\mathrm{p}=$ 0.02; and were discharged home earlier, mean difference -22.1 (CI $-42.1,-2.2$ ) days, $p=0.04$. There was no significant difference in the relative risk of any complication.

Conclusions-Trophic feeding improves clinical outcome in ill preterm infants requiring parenteral nutrition.

(Arch Dis Child Fetal Neonatal Ed 2000;82:F29-F33)

Intensive Care Unit,

St James's University

Hospital,

Leeds

R J McClure

S J Newell

Correspondence to:

Dr R J McClure,

Neonatal Services,

Rosie Hospital,

Addenbrooke's NHS Trust,

Cambridge CB2 2QQ

Email: robmcclure@msexc.

addenbrookes.anglox.nhs.uk

Accepted 23 June 1999 parenteral nutrition. Villi become shorter, mucosal DNA is lost, and protein content and enzymatic activity are reduced. ${ }^{1-6}$ In the rat model atrophy occurs after only three days of no enteric intake. ${ }^{5}$ Gastrointestinal atrophy and dysfunction are reversed following the introduction of enteral feeding. ${ }^{36}$

Trophic feeding (synonyms include minimal enteral feeding or nutrition, gut priming, and early hypocaloric feeding) is a relatively recent concept which has been introduced into clinical practice in an attempt to counter the effects of enteral starvation. It may be defined as the practice of feeding nutritionally insignificant volumes of enteral substrate to sick neonates, to supply nutrients to, and directly stimulate, the developing gastrointestinal system without increasing illness severity. Typically, milk volumes of $12-24 \mathrm{ml} / \mathrm{kg} /$ day are given. Several small randomised published studies (largest of which was 40 infants) have examined clinical outcome after trophic feeding. ${ }^{7-11}$ Individually they have suggested that milk tolerance, ${ }^{7-11}$ liver function, ${ }^{7}$ metabolic bone disease, ${ }^{7}$ days to hospital discharge, ${ }^{9}$ and weight gain ${ }^{11}$ are improved after trophic feeding. Systematic meta-analysis of these studies has revealed that only time to full enteral feeding, number of days that feedings were withheld, and total hospital stay were significantly reduced. ${ }^{12}$ Further critical appraisal of the clinical benefits trophic feeding in the high risk preterm infant seemed, therefore, to be warranted.

We performed a prospective randomised study of trophic feeding and clinical outcome in high risk preterm infants receiving intensive care. As changes in gastrointestinal function may have wide ranging effects a large number of clinical outcomes were studied, including energy intake growth, liver function, sepsis frequency, milk tolerance, incidence of selected complications and time to hospital discharge.

\section{Methods}

The study was performed on the neonatal intensive care unit at St James's University Hospital, Leeds. On day 3 of life preterm infants were consecutively recruited into the study if they satisfied all the following criteria: birthweight of less than $1750 \mathrm{~g}$; respiratory distress syndrome requiring mechanical ventilatory support beyond 48 hours; and they had received no previous enteral feeding. Infants were excluded if they had evidence of primary gastrointestinal pathology or a lethal congenital malformation. Infants left the study when discharged home, either from St James's or another hospital to which they had been transferred for further care. 
Informed parental consent was obtained before entry into the study, and the protocol was approved by the local research ethics committee.

Infants were stratified by gestation and then randomised into two groups using computer software (Minimisation program for allocating patients to treatments in clinical trials, The London Hospital Medical College). The TF group received trophic feeding and parenteral nutrition. Trophic feeding was started on day 3 at the rate of $0.5 \mathrm{ml} / \mathrm{h}$ if birthweight was less than $1 \mathrm{~kg}$, and $1 \mathrm{ml} / \mathrm{h}$ if birthweight was equal to or greater than $1 \mathrm{~kg}$. Trophic feeding continued at the same rate until mechanical ventilatory support was withdrawn. At this point "nutritive" milk feeds were introduced at a rate of $1 \mathrm{ml} / \mathrm{h}$ and then increased until full feeding was established. The rate of increase was determined by tolerance and was at the discretion of the attending nurse, but usually volume was increased by $1 \mathrm{ml}$ every $8-12$ hours.

Group C received parenteral nutrition alone until mechanical ventilatory support was no longer required. Nutritive milk feeding was then introduced at a rate of $1 \mathrm{ml} / \mathrm{h}$ and subsequently increased as for group $\mathrm{TF}$.

The mother chose at all times whether her expressed breast milk (EBM) or a preterm formula (Nutriprem, Cow \& Gate Nutricia) was fed to her infant. If EBM was unavailable then preterm formula was given. EBM was fortified with a commercial powdered preparation (Breast milk fortifier, Cow \& Gate Nutricia) once $90 \mathrm{ml} / \mathrm{kg} /$ day of milk was tolerated. All milk feeds were initially administered by hourly bolus via a nasogastric feeding tube.

The regimen of parenteral nutrition used for both groups was the same and consisted of a solution of amino acids (Vaminolact, Pharmacia-Upjohn), 10\% dextrose, electrolytes, trace minerals and vitamins, plus a fat emulsion (Intralipid 20\%, Pharmacia-Upjohn). Parenteral nutrition was started on day 2 of life and the content was incrementally increased to meet each infant's total estimated nutritional requirements by day 7 . The regimen of calorie and fluid increase was matched for both groups and took into account any contribution from trophic feeding. However, every effort was made to optimise the energy intake for infants in each group throughout the study.

No attempt was made to blind carers in the neonatal intensive care unit to infant group allocation. It was considered both impractical and unethical to pass and leave nasogastric feeding tubes in situ and give sham feeds of milk to infants in group C. However, staff caring for infants discharged to the special care unit or other hospitals as well as those measuring laboratory samples were all blinded to group allocation.

Total weekly energy intake was calculated, taking into account all parenteral and enteral nutrition received. Infants were compared over the first six postnatal weeks. Growth was assessed from birth to 6 weeks of postnatal age by performing weekly serial measurements of weight, head circumference, mid arm circumference (MAC) and, with the aid of a skin calliper (Holtain), triceps skinfold thickness. To

\section{Key messages}

- Timing of the introduction of milk feeds in sick low birthweight infants is controversial. Almost all infants with nonsurgical illness can tolerate at least some milk as trophic feeds.

- Trophic feeding leads to improved energy intake, weight gain, milk tolerance, less sepsis and earlier hospital discharge. No increase in major complication rate is seen following trophic feeding.

allow for different gestations at birth, the weekly increment was calculated for each anthropometric parameter and these data were used to compare groups.

To determine liver function, serum concentrations of conjugated bilirubin, alkaline phosphatase, and alanine transaminase were measured weekly in each infant. Serum total bilirubin concentration was also measured daily for the first 14 postnatal days and then weekly. The maximum value of each biochemical measurement of liver function for each infant was used for comparison. The total hours of phototherapy received were also recorded.

The incidence of sepsis was assessed in each group by recording the number of clinically septic episodes confirmed by microbial culture of blood, cerebrospinal fluid, or urine obtained by suprapubic aspiration. The total number of days when the $\mathrm{C}$ reactive protein was greater than 10 $\mathrm{mg} / \mathrm{l}$ (upper normal limit of local laboratory) was also recorded. C reactive protein is a sensitive non-specific acute phase protein that increases in serum concentration during episodes of sepsis. ${ }^{13}$ It was measured daily in infants receiving assisted ventilation and then later when sepsis was suspected.

Milk tolerance was assessed by recording days to toleration of full milk feeds (defined as $165 \mathrm{ml} / \mathrm{kg} /$ day), days to take full "sucking" feeds (full breast or bottle feeds), and days of parenteral nutrition. Each episode of the following complications was recorded for each infant: abdominal distension, bile stained vomiting or gastric aspirates, blood stained vomiting or gastric aspirates, necrotising enterocolitis (modified Bell's stage II or III), ${ }^{14}$ aspiration pneumonitis, and death. Required days of assisted ventilatory support, including continuous positive airways pressure, and supplemental oxygen were recorded. Finally, the total number of days between birth and first discharge home were recorded.

Data were collected directly from the relevant hospital records after hospital discharge. When required, personal visits were made by the authors to inspect the records of hospitals to which infants were transferred for further care. All biochemical and microbiological tests were performed by the local hospital departments of biochemistry and microbiology using standard methods. Data were analysed using the SPSS for windows statistical package version 8.0 (SPSS Inc, Chicago, USA). Linear 
regression analyses were used to assess the relation of the predictor variables of group randomisation, birthweight, CRIB score for assessing neonatal risk ${ }^{15}$ and days of postnatal dexamethasone to each clinical outcome. The mean difference with its $95 \%$ confidence interval was also calculated for each outcome, where appropriate. Growth parameters were compared by performing an analysis of covariance for each parameter gain, testing the effect of the classification factors of gender and group, while controlling for the covariates of birthweight ratio and gestation. The relative risk (event rate ratio) with $95 \%$ confidence interval was calculated for each complication.

\section{Results}

One hundred infants were studied. Forty eight were randomly allocated into group $\mathrm{TF}$ and 52 into group C. Six infants died in group TF, 11 in group C. Twenty one and 24 infants, respectively, from each group were transferred to another hospital before discharge home. Mean age at transfer was 29.5 days and 34.5 days for group $\mathrm{TF}$ and $\mathrm{C}$, respectively, mean difference -5 days $(-15.3,5.4), p=0.3$. Table 1 shows the baseline characteristics of the two groups. Infants in group $\mathrm{TF}$ received trophic feeding for a median (interquartile range) of 14 (7.5, 21.5) days. Trophic feeding was restricted or omitted on a total of 351 out of a possible 791 days. Reasons included abdominal distension, severe recurrent apnoea, bile stained gastric aspirates, milk pooling in the stomach and illness.

Energy intake over the first eight postnatal weeks is summarised in table 2. During the first six postnatal weeks infants in group $\mathrm{TF}$ received a significantly greater total energy intake than the control group, mean difference 41.4 (CI 9, 73.7) $\mathrm{kcal} / \mathrm{kg}$ (equivalent to 6.9 $\mathrm{kcal} / \mathrm{kg} /$ day or more over the whole 6 weeks). The significance level for group allocation, once controlled for the other predictor variables, was $p=0.02$. The coefficient value was -37.25 (SE 15.4) for the control group with group $\mathrm{TF}$ as the baseline.

The mean weekly gains in weight for each group are shown in fig 1 . The weight gain over 6 weeks was significantly greater in group $\mathrm{TF}$ than in group C, adjusted mean difference 130 g (CI 1, 250), p =0.02. Infants in group TF also had a significantly greater head circumference gain than those in group $\mathrm{C}$, adjusted mean difference $0.7 \mathrm{~cm}$ (CI $0.1,1.3$ ), p $=0.04$. Infants in group $\mathrm{TF}$ had a greater gain in both

Table 1 Baseline characteritics of studied infants

\begin{tabular}{lll}
\hline Variable & $\begin{array}{l}\text { Group TF } \\
(n=48)\end{array}$ & $\begin{array}{l}\text { Group } C \\
(n=52)\end{array}$ \\
\hline Male:female ratio & $1: 1.2$ & $1: 1$ \\
Gestation (completed weeks) & $28(27,30)$ & $28(26,30)$ \\
Birthweight (kg) & $1.1(0.9,1.4)$ & $0.94(0.8,1.3)$ \\
Birthweight ratio & $0.93(0.8,1.1)$ & $0.97(0.8,1.1)$ \\
CRIB score & $4(3,6.5)$ & $5(3,8)$ \\
$\begin{array}{l}\text { Antenatal steroids (doses) } \\
\text { Postnatal steroids (days) }\end{array}$ & $2(0,2)$ & $2(0,2)$ \\
$\begin{array}{c}\text { Breast milk:preterm formula } \\
\quad \text { milk ratio (majority feed) }\end{array}$ & $1: 0.7$ & $12.5(0,30)$ \\
\end{tabular}

Figures denote the median (interquartile range). $p$ value obtained using Mann-Witney U test and Fisher's exact test (indicated by ${ }^{\star}$ )
Table 2 Energy intake during the first 8 weeks

\begin{tabular}{lll}
\hline Week & Group TF & Group $C$ \\
\hline 1 & $72(65,78)$ & $69(61,77)$ \\
2 & $105(96,110)$ & $103(94,107)$ \\
3 & $113(102,130)$ & $102(89,121)$ \\
4 & $129(111,135)$ & $126(109,133)$ \\
5 & $134(120,135)$ & $132(117,135)$ \\
6 & $135(120,135)$ & $132(119,135)$ \\
7 & $135(132,135)$ & $133(130,135)$ \\
8 & $135(132,135)$ & $134(131,135)$ \\
Whole period of first & & \\
$\quad$ 6 postnatal weeks & $104(100,112)$ & $102(95,110)$ \\
Whole period of first & & \\
8 postnatal weeks & $130(122,140)$ & $130(119,138)$ \\
\hline
\end{tabular}

Figures denote median (interquartile range) daily $\mathrm{kcal} / \mathrm{kg}$ energy intake during each postnatal week.

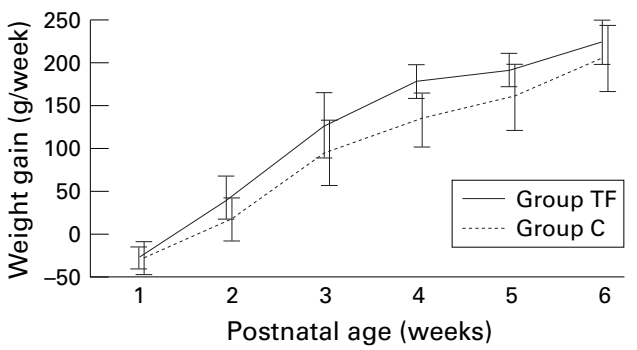

Figure 1 Mean weekly weight gain. Horizontal bars denote $95 \%$ confidence interval around the mean.

mid-arm circumference and triceps skin fold thickness as well, but the differences were not significant, adjusted mean difference $0.27 \mathrm{~cm}$ $(-0.2,0.7), \mathrm{p}=0.61$ and $0.25 \mathrm{~mm}(\mathrm{CI}-0.2$, $0.7), p=0.53$, respectively.

The mean values for the other clinical outcomes are shown in table 3 . There was no significant difference between groups for any measure of liver function including duration of jaundice.

Infants in group $\mathrm{TF}$ had significantly fewer episodes of culture confirmed sepsis, mean difference -0.7 (CI $-1.3,-0.2)$ septic episodes, $p$ $=0.04$, coefficient 0.5 (SE 0.2). Organisms cultured in group TF were coagulase negative staphylococci (18 episodes), enterococci species (2), Staphylococcus aureus (1), coliform species (1), $\alpha$ haemolytic streptococcus (1), and Aspergillus fumigatus (1). The increase in sepsis in group $\mathrm{C}$ was mainly attributable to coagulase negative staphylococci (45) and $S$ aureus (7). The other organisms were enterococci species (2), coliform species (3), A fumigatus (2), and Candida albicans (2). Infants in group $\mathrm{TF}$ also had fewer days when the serum $\mathrm{C}$ reactive protein concentration was greater than $10 \mathrm{mg} / \mathrm{l}$, mean difference -2.1 (CI -4 , -0.1 ) days, $\mathrm{p}<0.05$, coefficient 1.8 (SE 0.8).

Infants in group TF required significantly fewer mean days of parenteral nutrition than those in group $\mathrm{C}$, mean difference -11.5 (CI $-20,-3$ ) days, $\mathrm{p}=0.01$, coefficient 9.2 (SE 3.6). Infants in group $\mathrm{TF}$ also tolerated full milk feeds $(165 \mathrm{ml} / \mathrm{kg} /$ day $)$ significantly earlier, mean difference $-11.2(\mathrm{CI}-19,-3)$ days, $\mathrm{p}=$ 0.02 , coefficient 9 (SE 3.8).

Similarly, full "sucking" feeding was earlier in group TF but the difference was not significant, mean difference -15.6 (CI $-32,1)$ days, $\mathrm{p}=0.08$.

There was no difference in the mean requirement for assisted ventilation, mean dif- 
Table 3 Clinical outcomes

\begin{tabular}{|c|c|c|c|c|}
\hline Clinical outcome & Group $T F$ & Group $C$ & Mean difference & $p$ \\
\hline $\begin{array}{l}\text { Maximum serum conjugated bilirubin } \\
\text { concentration }(\mu \mathrm{mol} / \mathrm{l})\end{array}$ & $28(23,33)$ & $30(23,38)$ & $-2(-12,7)$ & 0.6 \\
\hline $\begin{array}{l}\text { Maximum serum total bilirubin } \\
\text { concentration }(\mu \mathrm{mol} / \mathrm{l})\end{array}$ & $215(201,229)$ & $207(190,225)$ & $7.5(-15,30)$ & 0.5 \\
\hline $\begin{array}{l}\text { Maximum serum alkaline phosphatase } \\
\text { concentration (IU/l) }\end{array}$ & $975(809,1141)$ & $915(782,1048)$ & $60(-149,269)$ & 0.6 \\
\hline Maximum serum alanine transaminase & & & & \\
\hline concentration (IU/l) & $28(21,36)$ & $41(25,57)$ & $-13(-30,4)$ & 0.1 \\
\hline Phototherapy (hours) & $56(44,67)$ & $44(32,56)$ & $12(-5,28)$ & 0.2 \\
\hline Duration of jaundice (days) & $32(26,38)$ & $31(24,37)$ & $1.3(-7.1,9.7)$ & 0.5 \\
\hline Culture confirmed sepsis (episodes) & $0.5(0.3,0.7)$ & $1.2(0.7,1.7)$ & $-0.7(-1.3,-0.2)$ & $0.04 \dagger$ \\
\hline C reactive protein $>10 \mathrm{mg} / 1$ (days) & $2.2(1,3.4)$ & $4.3(2.8,5.8)$ & $-2.1(-4,-0.1)$ & $<0.05 \dagger$ \\
\hline PN (days) & $20.6(17,25)$ & $32.1(24,39)$ & $-11.5(-20,-3)$ & $0.01 \dagger^{\circ}$ \\
\hline Time to full enteral feeds (days) & $24.8(21,29)$ & $36.1(28,44)$ & $-11.2(-19,-3)$ & $0.02 \dagger$ \\
\hline Time to full "sucking" feeds (days) & $61.5(54,69)$ & $77.1(61,93)$ & $-15.6(-32,1)$ & 0.08 \\
\hline Assisted ventilation (days) & $14.1(11,17.2)$ & $20.2(12.2,28.1)$ & $-6.1(-14.8,2.6)$ & 0.2 \\
\hline Supplemental oxygen (days) & $24(17.4,30.7)$ & $46.4(29,63.9)$ & $-22.4(-41.5,-3.3)$ & $0.02 \dagger$ \\
\hline Hospital discharge (days) & $70.3(61.8,78.9)$ & $92.4(73.9,111)$ & $-22.1(-42.1,-2.2)$ & $0.04 \dagger$ \\
\hline
\end{tabular}

All outcomes are expressed as mean values with $95 \%$ confidence interval in parentheses.

†Denotes statistical significance.

ference -6.1 (CI $-14.8,2.6$ ) days, $\mathrm{p}=0.2$. However, infants in group TF required significantly fewer mean days of supplemental oxygen than those in group C, mean difference -22.4 (CI $-41.5,-3.3)$ days, $\mathrm{p}=0.02$, coefficient 25.3 (SE 10.2).

The total number and relative risk of each recorded complication are shown in table 4 . There was no significant difference in the relative risk for any complication.

Infants in group $M$ were discharged home after a mean of 70.3 (CI 61.8, 78.9) days compared with 92.4 (CI 73.9, 111) days in group C. This difference was significant, mean difference $-22.1(-42.1,-2.2)$ days, $p=0.04$, coefficient 14.8 (SE 7.2).

\section{Discussion}

There has been a recent shift in neonatal practice towards the greater use of enteral feeds in ill preterm neonates. ${ }^{16}$ This move has to some extent been influenced by the increase in the use of enteral feeds in paediatric and adult intensive care units. The findings of this study support this current trend. The infants exposed to trophic feeding had significantly greater energy intake, greater weight gain and head growth, improved milk tolerance, less requirement for parenteral nutrition, less sepsis, fewer days of supplemental oxygen and were discharged from hospital earlier than those that did not. No adverse effect was detected. This was despite the considerable shortfall in trophic feeding from that intended, reflecting the illness severity of the infants studied. Although the regimen for increasing energy intake was the same for both groups, infants were not matched. We considered it unethical to restrict energy intake for any infant solely for

Table 4 Total episodes and relative risks for complications

\begin{tabular}{llcl}
\hline Complication & Group TF & GroupC & $\begin{array}{l}\text { Relative risk } \\
\text { (95\% CI) }\end{array}$ \\
\hline Abdominal distension & 11 & 7 & $1.6(0.7,3.9)$ \\
Bile stained gastric aspirates/vomits & 27 & 25 & $1.8(0.8,1.7)$ \\
Blood stained gastric aspirates/vomits & 3 & 8 & $0.4(0.1,1.4)$ \\
Necrotising enterocolitis & 1 & 2 & $0.6(0.1,5.9)$ \\
Aspiration pneumonia & 0 & 0 & 1 \\
Death & 6 & 11 & $0.6(0.2,1.5)$ \\
\hline
\end{tabular}

Values expressed are actual total number of episodes. $95 \%$ confidence intervals are stated in parentheses. research purposes. Whether the greater energy intake following trophic feeding was responsible for the various favourable clinical outcomes and growth, or was a consequence of them, is unclear, and, is perhaps, an academic question. However, even assuming all the total median $290 \mathrm{kcal} / \mathrm{kg}$ extra received over six weeks by infants in group TF was absorbed, this amount would probably be insufficient to account for all of the extra mean $130 \mathrm{~g}$ weight gain enjoyed by these infants during this period.

There was no difference in the indicators of liver function or need for phototherapy. This does not accord with the findings of Dunn et $a l,{ }^{7}$ but was similar to the observations of Slagle and Gross and Meetze et al. ${ }^{89}$ As both the later studies, and this study, were larger than that of Dunn et $a l$, it is reasonable to conclude that liver function is not altered by trophic feeding even if parenteral nutrition requirement is reduced.

A reduction in both culture confirmed sepsis and a marker of sepsis was seen following trophic feeding. Nosocomial infections result in higher infant mortality and morbidity, prolonged inpatient stay and increased costs. ${ }^{17-19}$ Sepsis may have been reduced secondary to the decreased need for parenteral nutrition. The large difference in episodes of coagulase negative staphylococci seem to suggest this. Parenteral nutrition predisposes to sepsis because of both its interference with the immune system and the potential portal of infection via intravenous cannula. ${ }^{20}{ }^{21}$ Alternatively, translocation of enteric pathogenic micro-organisms into the circulation may have been reduced either because of improved gastrointestinal mucosal barrier function or beneficial alteration of the enteric flora.

The significant reduction in parenteral nutrition requirement and time to full milk feeds support the findings of previous studies that trophic feeding improves later milk tolerance..$^{7-11} \mathrm{~A}$ mean reduction of over 11 days in parenteral nutrition requirement, as found in this study, should significantly reduce the associated morbidity with this treatment, as well as provide considerable economic savings. At the time of writing parenteral nutrition costs 
$£ 30$ and intensive care over $£ 700$ per day on the unit where the study was conducted.

This study did not have the power to determine anything other than a major association between trophic feeding and a severe complication such as death or necrotising enterocolitis. It is unreasonable, therefore, to conclude that trophic feeding is without risk. However, infants given trophic feeding did seem to have more minor gastrointestinal complications (abdominal distension, bile stained vomiting or gastric aspirates) than the controls. Reassuringly, the number of infants with necrotising enterocolitis, aspiration pneumonia, or, most importantly, death were not more numerous in the group given trophic feeding.

It was perhaps surprising that supplemental oxygen requirement was less in those infants given trophic feeding. Respiratory outcomes were not primary outcome measures of this study. This would have required controlling infants for severity of initial respiratory disease. It is, therefore, unclear whether the intriguing apparent improvement in respiratory status was genuine. A true effect might have been the result of improved energy intake and growth, decreased sepsis, and parenteral nutrition, or agents present in milk which are beneficial to the lung.

As so many outcomes were affected the benefits of trophic feeding are likely to have been mediated by more than one mechanism. Plausible mechanisms include: greater energy intake; the presence of agents in milk that are trophic to the gastrointestinal tract, such as glutamine, arginine or insulin-like growth factors ${ }^{22}$; induction of gastrointestinal motor activity development ${ }^{9}$; the release of trophic enteric hormones ${ }^{93}$; and induced physiological changes in the enteric mucosa via stimulation of specific local surface receptors by nutrients. The mechanisms of action remain poorly defined, but the benefits of trophic feeding shown by this study strongly support its use for the preterm infant requiring intensive care and parenteral nutrition.

This work was generously supported by a grant from the Nutricia Research Foundation. We are indebted to the staff members of the regional neonatal intensive care unit at St James's University Hospital, Leeds, for their help and cooperation, and to D PRF Dear for his advice and support.

1 Levine GN, Deren JJ, Steiger E, Zinno R. Role of oral intake in maintenance of gut mass and disaccharide activity. Gastroenterology 1974;67:975-82.
2 Feldman E, Dowling R, McNaughton J, Peters T. Effects of oral versus intravenous nutrition on intestinal adaptation after small bowel resection in the dog. Gastroenterology
1974;70:712-9.

3 Greene HL, McCabe DR, Merenstein GB. Protracted diarrhea and malnutrition in infancy: Changes in intestinal morphology and disaccharidase activities during treatment with total intravenous nutrition or oral elemental diets. $\mathcal{F}$ Pediatr 1975;87:695-704.

4 Dworkin LD, Levin GM, Farber NJ, Spector MH. Small intestinal mass of the rat is partially determined by indirect effects of intraluminal nutrition. Gastroenterology 1976;71:626-30.

5 Hughes CA, Dowling RH. Speed of onset of adaptive mucosal hypoplasia and hypofunction in the intestine of parenterally fed rats. Clin Sci 1980;59:317-27.

6 Pironi L, Paganelli GM, Miglioli M, Biasco G, Santucci R, Ruggeri E. Morphologic and cytoproliferative patterns of duodenal mucosa in two patients after long-term total parenteral nutrition: changes with oral refeeding and relation to intestinal resection. I Parent Ent Nutr 1994;18:351-4

7 Dunn L, Hulman S, Weiner J, Kliegman R. Beneficial effects of early enteral feeding on neonatal gastrointestinal function: Preliminary report of a randomised trial. $\mathcal{F}$ Pediatr 1988;112:622-9.

8 Slagle TA, Gross SJ. Effect of early low-volume enteral substrate on subsequent feeding tolerance in very low birth weight infants. F Pediatr 1988;113:526-31.

9 Berseth CL. Effect of early feeding on maturation of the preterm infant's small intestine. F Pediatr 1992;120:94753.

10 Meetze WH, Valentine C, McGuigan JE, Conlon M, Sacks $\mathrm{N}$, Neu J. Gastrointestinal priming prior to full enteral nutrition in very low birth weight infants. $\mathcal{F}$ Pediatr Gastroenterol Nutr 1992;15:163-70

11 Troche B, Harvey-Wilkes K, Engle WD, et al. Early minimal feedings promote growth in critically ill premature infants. Biol Neonate 1995;67:172-181.

12 Tyson JE, Kennedy KA. Minimal enteral nutrition in parenterally fed neonates. In: Sinclair JC, Bracken MB, Soll RF, Horbar JD, eds. Neonatal module of The Cochrane Database of Systematic Reviews. Oxford: 1997.

13 Russell GA, Smyth A, Cooke RW. Receiver operating characteristic curves for comparison of serial neutrophil band
forms and $\mathrm{C}$ reactive protein in neonates at risk of infection. Arch Dis Child 1992;67:808-12.

14 Walsh MC, Kliegman RM. Necrotizing enterocolitis: Treatment based on staging criteria. Pediatr Clin North Am 1986;33:79-201.

15 The International Neonatal Network. The CRIB (clinical risk index for babies) score: a tool for assessing initial neonatal risk and comparing performance of neonatal intensive care units. Lancet 1993;342:193-8.

16 McClure RJ, Chatrath MR, Newell SJ. A survey of the variety and changing trends in feeding policies for ventilated pre-term infants in the United Kingdom. Acta Paediatr 1996;85:1123-5.

17 Hemming VG, Overall JC, Jr., Britt MR. Nosocomial infections in a newborn intensive care unit. Results of forty-one months of surveillance. N Engl f Med 1976;294:1310-16.

18 LaGamma EF, Drusin LM, Mackles AW, Machalek S, Auld PAM. Neonatal infections: An important determinant of late NICU mortality in infants less than $1000 \mathrm{~g}$ at birth. Am $\mathcal{F}$ Dis Child 1983;137:838-41.

19 Girard R, Fabry J, Meynet R, Lambert DC, Sepetjan M. Costs of nosocomial infection in a neonatal unit. f Hosp Infect 1983;4:361-6.

20 Yu VYH, James B, Hendry P, MacMahon RA. Total parenteral nutrition in very low birthweight infants: a controlled trial. Arch Dis Child 1979;54:653-61.

21 Unger A, Goetzman BW, Chan C, Lyons AB, Miller MF. Nutritional practices and outcome of extremely premature infants. Am $\mathcal{F}$ Dis Child 1986;140:1027-33.

22 Carver JD, Barness LA. Trophic factors for the gastrointestinal tract. Clin Perinatol 1996;23:265-85.

23 Lucas A, Bloom SR, Aynsley-Green A. Gut hormones and "minimal enteral feedings". Acta Paediatr Scand 1986;75:719-23. 